\title{
Machian derivation of the Friedmann equation
}

\author{
Herman Telkamp \\ Jan van Beverwijckstraat 104, 5017 JA Tilburg, The Netherland * $^{2}$
}

(Dated: June 9, 2016)

\begin{abstract}
Despite all fundamental objections against Newtonian concepts in cosmology, the Friedmann equation derives from these in an astoundingly simple way through application of the shell theorem and conservation of Newtonian energy in an infinite universe. However, Friedmann universes in general posses a finite gravitational horizon, as a result of which the application of the shell theorem fails and the Newtonian derivation collapses. We show that in the presence of a gravitational horizon the Friedmann equation can be derived from a Machian definition of kinetic energy, without invoking the shell theorem. Whereas in the Newtonian case total energy translates to curvature energy density, in the Machian case total energy takes on different identities, depending on the evolution of the horizon; we show that in the de Sitter universe Machian total energy density is constant, i.e. appears as cosmological constant.
\end{abstract}

PACS numbers: 98.80.Jk 95.36.+x 04.50.Kd

\section{INTRODUCTION: NEWTONIAN DERIVATION OF THE FRIEDMANN EQUATION}

The Friedmann equation, describing the evolution of the scale factor $a$ of the expanding universe, reads

$$
\frac{\dot{a}^{2}}{a^{2}}=\frac{8}{3} \pi G\left(\rho+\rho_{k}\right),
$$

where, according general relativity (GR), $\rho$ is the total density of the various matter sources and where $\rho_{k} \propto a^{-2}$ represents curvature energy.

The density of pressureless matter (dust) causes deceleration of expansion, which is commonly attributed to the attractive force of gravity between cosmic masses. This view on pressureless matter, i.e., deceleration by attraction, is supported by the well known Newtonian cosmology (see e.g. [1, 2]), which involves both the shell theorem (the field within an empty spherical mass shell is uniformly zero) and the conservation of energy, $E=T+V$, of a test mass $m$ at the edge of an arbitrary spherical volume of the universe, where $T$ and $V$ represent Newtonian kinetic and potential energy of $m$ relative to (the center of) this sphere. For a spherical volume of proper radius $R_{s}=a \chi_{s}$, where $\chi_{s}$ is the constant comoving coordinate, this straightforwardly leads [1] to the Newtonian energy conservation equation

$$
T+V=\frac{1}{2} m \dot{a}^{2} \chi_{s}^{2}-\frac{4}{3} m \pi G \rho a^{2} \chi_{s}^{2}=E=\text { const. }
$$

which is precisely the Friedmann equation (1); $\chi_{s}$ is fixed, so constant total energy $E$ indeed translates to curvature density, $\rho_{k} \propto E / a^{2} \chi_{s}^{2}$, as can be verified easily. In a strict Newtonian sense the matter density $\rho$ regards ordinary pressureless matter only, but for the Newtonian line of

\footnotetext{
* herman_telkamp@hotmail.com
}

thought there is no objection to also include other matter sources, like this is essential in GR.

We note that Newton was surprisingly successful as the same equation was derived by Friedmann from GR (which derivation is actually much more involved, yet physically sound). What is bothering though is that, considering all fundamental objections against Newtonian cosmology, the Newtonian result is almost too good to be true. Not only the result is consistent with the GR derivation, the Newtonian derivation also shows that, with the simplest of arguments, classical concepts of kinetic, potential and conserved total energy still seem to make sense, while these are often believed not to hold globally in (relativistic) cosmology. Perhaps the Newtonian idea of conservation of energy in the universe seemingly at war with GR - deserves more credit than being educational alone.

On the other hand, Newtonian concepts like infinite speed of gravity and absolute space are generally considered unphysical. We therefore assume gravity propagating at the speed of light, along with the presence of a gravitational cosmic horizon, which is the case with general Friedmann universes. However, as we will note, the application of the shell theorem fails in the presence of a horizon. And so does the Newtonian derivation. Instead, we consider purely relational (Machian) definitions of energy, due to Schrödinger [3]. Applying these, we arrive at a Machian derivation of the Friedmann equation by assuming conservation of total Machian energy, and without invoking the shell theorem. This derivation holds, like in the GR case, for general Friedmann universes, with or without a horizon.

\section{LIMITATIONS AND INCONSISTENCY OF THE NEWTONIAN DERIVATION}

Though intuitive (matter attracts other matter), Newtonian cosmology rests on the dubious assumptions of instantaneous "action at a distance" in an infinite un- 
bounded universe. Within GR however, Friedmann universes in general posses a gravitational horizon, due to propagation of gravity at the speed of light in a universe of finite age. We therefore assume a propagating gravitational horizon at comoving distance $\chi_{g}$.

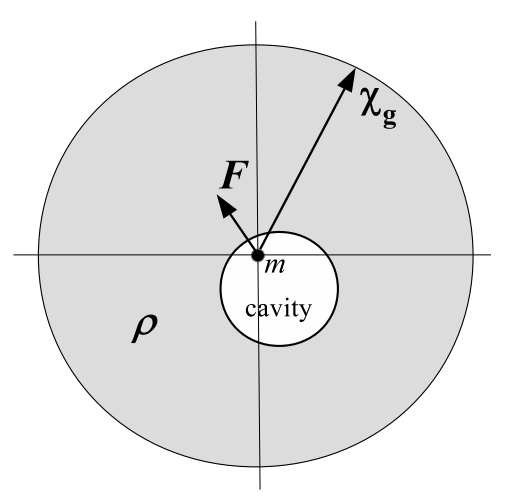

FIG. 1. Gravitational force $F$ inside an empty spherical cavity within the horizon $\chi_{g}$.

The whole argument of Newton's derivation is based on the assumption that the mass distribution outside an empty spherical cavity of the universe is spherically symmetric, so that the shell theorem applies and the field inside the cavity is uniformly zero. This assumption holds in an infinite homogeneous isotropic universe. However, the presence of a gravitational horizon, centered at an observer somewhere in the cavity, breaks this symmetry; due to the introduction of a horizon the field inside the cavity is no longer uniformly zero, as is clear from Fig 1 the asymmetry causes a non-zero field directed away from the center of the cavity.

As a result the Newtonian derivation collapses for universes bounded by a gravitational horizon, which, ironically, is the case with general Friedmann universes. And actually, one would not expect differently, since due to symmetry the gravitational field in the perfectly homogeneous isotropic universe is zero everywhere, with or without a horizon. This makes the concept of deceleration by gravitational attraction questionable. For the same reason one must question the concept of a "repulsive force" due to vacuum energy.

With no forces acting, what then is left to even explain deceleration in a Newtonian sense? The zero field points at constancy of the kinetic energy of recession; no energy is being exchanged. In a Newtonian context, where mass inertia is invariant, constant kinetic energy implies constant recession velocities, i.e., a coasting universe, as in Melia [4]. Hence, if Newtonian physics predicts anything, then it is a coasting universe, rather than a decelerating universe; a conclusion earlier arrived at e.g. by Layzer [5].

\section{MACHIAN PHYSICS}

The Machian approach due to Schrödinger [3] shares features of Newtonian mechanics, however defined in a purely relational framework, by which it considers causal relations of all matter within the cosmological horizon. Such an explicit treatment, as pursued hereafter, discerns the Machian approach from both Newton's and Einstein's theory. In a Machian context mass inertia is a relational property which depends on the "distribution of matter", and so inertia is likely to evolve in an expanding universe with a cosmological horizon. Like in GR, and following Schrödinger approach in his Machian derivation of the anomalous perihelion precession [3], we assume the influence of the matter sources is through the gravitational potential. That is, we assume the inertia of a point particle to depend on the gravitational potentials of all other interacting point particles, in a one-on-one fashion. By integration of the elementary point particle relations one can derive expressions for the interaction of arbitrary finite bodies, like e.g. two solid spheres, or (as in our case) a small test particle relative to the surrounding sphere of cosmic matter, as explained below.

How to actually determine (cosmic) gravitational energy is a matter still open to debate. But given the surprising success of Newtonian cosmology, we assume a Newtonian potential. Though, we do allow the density parameter $\rho$ to include the various matter sources. We assume a homogeneous and isotropic universe. Different from Newton's derivation, we consider test particle $m$ at the center of the cosmic sphere of comoving radius $\chi_{g}$, thus regard all matter causally connected with $m$. The infinitesimal Newtonian potential at the origin due to the cosmic matter element $\mathrm{d}^{3} M$ at spherical coordinates $(\chi, \theta, \phi)$ is

$$
\mathrm{d}^{3} \varphi(\chi, \theta, \phi)=\frac{-G \mathrm{~d}^{3} M}{a \chi}=-G \rho a^{2} \chi \sin \theta \mathrm{d} \phi \mathrm{d} \theta \mathrm{d} \chi .
$$

Then the Newtonian cosmic potential due to all matter within the horizon is

$$
\varphi=\int_{0}^{\chi_{g}} \int_{0}^{\pi} \int_{0}^{2 \pi} \mathrm{d}^{3} \varphi=-2 \pi G \rho a^{2} \chi_{g}^{2} .
$$

This expression contrasts with Sciama [6], who derived, by the gravitoelectromagnetic analog of Maxwell's equations, a cosmic potential equal to

$$
\varphi_{u}=-c^{2} .
$$

Remarkably, the potential $\varphi_{u}$ is independent of any cosmic parameter. But as Sciama noted, it has to be like that, given that in our inertial frame Newton's laws hold without any reference to the cosmic masses. It is tempting to assume both potentials to be equal, $\varphi=\varphi_{u}$, but it is unnecessary for the present derivation of the Friedmann equation to make such an assumption.

Note that the Newtonian gravitational potential is genuinely Machian by its relational, frame independent 
nature. Newtonian kinetic energy, on the other hand, is frame dependent, thus not relational, so clearly not Machian. There is no established definition of Machian kinetic energy. Therefore, before considering Machian kinetic energy of the expanding universe, we will briefly introduce Machian physics according to the approach taken by Schrödinger in his Machian derivation of the anomalous perihelion precession [3]:

The main conceptual difference with Newtonian physics is that in Machian physics according Schrödinger inertia and kinetic energy are not intrinsic properties of a particle, but are mutual properties which arise from the interaction of the particle with all other (causally connected) particles, just like this is true for potential energy and the force of gravity. For the pair of point particles $m_{i}$ and $m_{j}$ we consider a frame independent definition of their mutual Machian kinetic energy, according

$$
T_{i j} \equiv \frac{1}{2} \mu_{i j} \dot{r}_{i j}^{2},
$$

where $r_{i j}$ denotes the proper radial distance (separation) of the particles. Crucial here is that from a relational point of view only radial motion is meaningful in the physical relationship of the two point particles. This ontological notion (epistemological if you like) is due to Bishop Berkeley [7], one of the earliest critics of Newton, who first pointed out that any motion between two point particles, other than their relative radial motion, in otherwise empty space is unobservable, therefore physically meaningless, or inexistent for that matter. Thus, two particles in circular orbit of each other have zero mutual kinetic energy between them. Each particle does however have mutual kinetic energy relative to the surrounding cosmic particles, namely proportional to the (square of the) radial component of motion towards each cosmic particle. Thus Machian physics is intimately connected with all the cosmic matter.

One can picture Machian kinetic energy between two particles as the energy that would be dissipated if one would freeze the relative motion between the particles. Fixing the separation of two spheres which are in elliptic orbit would definitely affect kinetic energy, but only as far as the radial component of motion is concerned (this also explains why the anomalous perihelion precession depends on the amount of eccentricity of the ellipse). To the contrary, fixing the distance between two spheres in perfect circular orbit would not affect energies at all; their mutual (Machian) kinetic energy is zero, just as Berkeley argued. So, if there is zero kinetic energy between spheres in perfect circular orbit, then the orbital kinetic energy of these two spheres must be entirely due to the presence of cosmic matter. That is, due to the motion of each sphere in or from the direction of surrounding cosmic matter.

Like kinetic energy $T_{i j}$, Machian inertia $\mu_{i j}$ is a relational and mutual property between any pair of point particles and is defined as

$$
\mu_{i j} \equiv m_{i} \frac{\varphi_{j}\left(r_{i j}\right)}{\varphi_{\text {eff }}}=m_{j} \frac{\varphi_{i}\left(r_{i j}\right)}{\varphi_{e f f}}=\frac{-G m_{i} m_{j}}{\varphi_{\text {eff }} r_{i j}},
$$

where the effective potential

$$
\varphi_{\text {eff }}=\frac{1}{3} \varphi
$$

serves as a normalization parameter which preserves consistency with Newtonian inertia, as shown below. The reason that a factor $\frac{1}{3}$ of the cosmic potential $\varphi$ appears (see Schrödinger [3]) is that in a Machian sense only the radial component of motion counts, i.e., the two perpendicular components of motion do not contribute. Hence, in any peculiar motion effectively only one third of the total cosmic potential contributes to the kinetic energy between a mass $m$ and the universe (see appendix $\mathrm{A}$ for details).

Of course one can argue whether the above Machian definitions are correct. Obviously they are no subtitute of GR. Yet, the underlying physical concepts (observability, causal connection of all matter within the cosmic horizon, abandoning absolute space, mutuality of both inertia and kinetic energy) are ontologically as good as irrefutable. But also the precise form of the definitions Eqs. (617) appears sensible. Inertia between two point particles is defined as their mutual potential energy and so expresses the equivalence of mass and energy. From these same definitions Schrödinger straightforwardly reproduced the GR expression of the anomalous perihelion precession. Since the Machian approach explicitly considers the causal connection of all matter within the gravitational cosmic horizon, it may provide a useful alternative in the physical interpretation of cosmology, like in the derivation of the Friedmann equation hereafter.

\section{MACHIAN DERIVATION OF THE FRIEDMANN EQUATION}

Using the above elementary definitions [Eqs. (6/7]8)], one can formulate the kinetic energy between a small mass $m$ at the origin and all receding matter within the gravitational horizon $\chi_{g}$. We assume (only initially) particle $m$ has a peculiar velocity $v$. This to evaluate consistency of the Machian definitions with Newtonian physics. For simplicity and without loss of generality we assume this peculiar velocity is in the polar direction $(\theta=0)$. The radial velocity $\dot{r}$ between $m$ and cosmic matter element $\mathrm{d}^{3} M$ at coordinates $(\chi, \theta, \phi)$ is thus

$$
\dot{r}=\chi \dot{a}-v \cos \theta .
$$

According Eq. (17) the mutual inertia between $m$ and matter element $\mathrm{d}^{3} M$ is, using Eqs. (34),

$$
\mathrm{d}^{3} \mu=m \frac{\mathrm{d}^{3} \varphi(\chi, \theta, \phi)}{\frac{1}{3} \varphi\left(\chi_{g}, a\right)}=m \frac{3 \chi \sin \theta}{2 \pi \chi_{g}^{2}} \mathrm{~d} \phi \mathrm{d} \theta \mathrm{d} \chi .
$$

By definition Eq. (6) the kinetic energy between particle $m$ and the cosmic matter element $\mathrm{d}^{3} M$ is 


$$
\begin{aligned}
\mathrm{d}^{3} T(\chi, \theta, \phi) & =\frac{1}{2} \mathrm{~d}^{3} \mu \dot{r}^{2} \\
& =m \frac{3 \chi \sin \theta}{4 \pi \chi_{g}^{2}}(\dot{a} \chi-v \cos \theta)^{2} \mathrm{~d} \phi \mathrm{d} \theta \mathrm{d} \chi
\end{aligned}
$$

Then the total Machian kinetic energy between the particle $m$ and all the cosmic matter within the horizon follows with some math (see appendix Arom the integral

$$
\begin{aligned}
T & =\frac{3}{4 \pi} \frac{m}{\chi_{g}^{2}} \int_{0}^{\chi_{g}} \int_{0}^{\pi} \int_{0}^{2 \pi} \chi \sin \theta(\dot{a} \chi-v \cos \theta)^{2} \mathrm{~d} \phi \mathrm{d} \theta \mathrm{d} \chi \\
& =\frac{3}{4} m \dot{a}^{2} \chi_{g}^{2}+\frac{1}{2} m v^{2} .
\end{aligned}
$$

Thus one obtains two distinct energies: recessional and peculiar kinetic energy. The cross term of peculiar motion and recession vanishes due to symmetry. We see that the peculiar part of the kinetic energy satisfies the Newtonian definition and that the Newtonian inertia $(m)$ is being retrieved. This means that the equivalence principle is maintained in peculiar motion, regardless of cosmic evolution. Hence, the Machian principle remains unnoticed in the local frame. This is consistent with the Hughes-Drever experiments [8] (a null result of anisotropy of inertia) and in agreement with both Dicke [9] and Sciama [6]. Indeed, Newtonian physics makes no reference to cosmological parameters, even though it derives here from a cosmological context.

Confining ourselves again to recessional motion only, we consider a unit test mass $m$ at rest in the Hubble flow $(v=0)$, and drop the symbol $m$ for simplicity. The potential energy of the particle is $V=\varphi$ [Eq. (4)], thus together with recessional kinetic energy $T=\frac{3}{4} \chi_{g}^{2} \dot{a}^{2}$ we have the Machian energy equation

$$
\frac{3}{4} \chi_{g}^{2} \dot{a}^{2}-2 \pi G \rho a^{2} \chi_{g}^{2}=E .
$$

Similar to the translation of total energy $E$ to curvature energy density $\rho_{k}$ in the Newtonian case, we introduce the (Machian) total energy density parameter

$$
\rho_{E}=E / 2 \pi G a^{2} \chi_{g}^{2}
$$

which, interestingly, is not necessarily curvature energy density, as $\chi_{g}$ in general evolves with the scale factor $a$ (see next section). Eq. (13) can now be rewritten

$$
\frac{3}{4} \chi_{g}^{2} \dot{a}^{2}=2 \pi G\left(\rho+\rho_{E}\right) a^{2} \chi_{g}^{2}
$$

Eliminating the common factor $\chi_{g}^{2}$, we obtain the Friedmann equation

$$
\frac{\dot{a}^{2}}{a^{2}}=\frac{8}{3} \pi G\left(\rho+\rho_{E}\right)
$$

\section{THE IDENTITY OF MACHIAN TOTAL ENERGY DENSITY}

The Machian energy equation Eq. (13) is nearly identical to the Newtonian version Eq. (2), however with a notable difference: $\chi_{s}$ is an arbitrary fixed comoving radius, while $\chi_{g}$ is (in general) an evolving gravitational horizon, $\chi_{g}=\chi_{g}(a)$. Thus in the Newtonian case total energy $E$ appears as curvature energy density always, i.e., $\rho_{k} \propto a^{-2} \chi_{s}^{-2}$, while in the Machian case total energy density in general also evolves with the horizon, $\rho_{E} \propto a^{-2} \chi_{g}^{-2}(a)$. Therefore, quite intriguingly, $\rho_{E}$ may take identities different from curvature energy, depending on the particular evolution of the horizon. For instance, in the de Sitter universe, and so in the late phase of the $\Lambda \mathrm{CDM}$ model, the proper distance to the event horizon is constant, hence $\chi_{g} \propto a^{-1}$. This translates total energy density to constant vacuum energy density, i.e., $\rho_{E}=\rho_{\Lambda}=$ const. Thus Machian cosmology potentially gives physical interpretation to the origin of the cosmological constant.

\section{CONCLUSION}

We argued that application of the shell theorem fails with universes bounded by a gravitational horizon, like general Friedmann universes are. Consequently the Newtonian derivation of the Friedmann equation collapses in the presence of a horizon, while the GR derivation holds for general Friedmann universes. We showed that, alternatively, the Friedmann equation can be derived from Machian arguments: for arbitrary homogeneous isotropic universes, either with or without a horizon, the Friedmann equation follows from conservation of a Machian definition of energy, without invoking the shell theorem. We further showed that, depending on the evolution of the horizon, the Machian total energy density takes different identities. In particular in a de Sitter universe, Machian total energy density appears as constant vacuum energy density, thus provides interpretation to the cosmological constant.

\section{Appendix A:}

Calculation details of the integral Eq. (12), i.e., of the Machian kinetic energy between a test mass $m$ at the origin and all receding matter within the cosmic horizon $\chi_{g}$, where $m$ is assumed to have a peculiar velocity $v$ in the polar direction $\theta=0$ : 


$$
\begin{aligned}
T= & \frac{3}{4 \pi} \frac{m}{\chi_{g}^{2}} \int_{0}^{\chi_{g}} \int_{0}^{\pi} \int_{0}^{2 \pi} \chi \sin \theta(\dot{a} \chi-v \cos \theta)^{2} \mathrm{~d} \phi \mathrm{d} \theta \mathrm{d} \chi \\
= & \frac{3}{2} \frac{m}{\chi_{g}^{2}} \int_{0}^{\chi_{g}} \int_{0}^{\pi} \dot{a}^{2} \chi^{3} \sin \theta-2 \dot{a} \chi^{2} v \sin \theta \cos \theta \\
& +\chi v^{2} \sin \theta \cos ^{2} \theta \mathrm{d} \theta \mathrm{d} \chi \\
= & \frac{3}{2} \frac{m}{\chi_{g}^{2}} \int_{0}^{\chi_{g}}\left[-\cos \theta \dot{a}^{2} \chi^{3}+0-\frac{1}{3} \chi v^{2} \cos ^{3} \theta\right]_{0}^{\pi} \mathrm{d} \chi \\
= & \frac{3}{2} \frac{m}{\chi_{g}^{2}} \int_{0}^{\chi_{g}} 2 \dot{a}^{2} \chi^{3}+\frac{2}{3} \chi v^{2} \mathrm{~d} \chi \\
= & \frac{3}{2} \frac{m}{\chi_{g}^{2}}\left[\frac{1}{2} \dot{a}^{2} \chi^{4}+\frac{1}{3} \chi^{2} v^{2}\right]_{0}^{\chi_{g}} \\
= & \frac{3}{4} m \dot{a}^{2} \chi_{g}^{2}+\frac{1}{2} m v^{2} .
\end{aligned}
$$

Since only the radial component of motion $(\dot{r}=\dot{a} \chi-$ $v \cos \theta)$ counts, the potential due to the cosmic matter element at coordinates $(\chi, \theta, \phi)$ contributes to the peculiar (i.e., Newtonian) kinetic energy of the object $m$ only by a fraction $\cos ^{2} \theta$. On average, i.e., in the spherical integral Eq. (A1), this fraction gives rise to a factor

$$
\frac{\int_{0}^{\pi} \sin \theta \cos ^{2} \theta d \theta}{\int_{0}^{\pi} \sin \theta d \theta}=\frac{1}{3} .
$$

Thus the effective contribution of the cosmic potential to the peculiar inertia is only $\varphi_{\text {eff }}=\frac{1}{3} \varphi$ [Eq. (8)].
[1] A. Liddle, An Introduction to Modern Cosmology (Wiley, 2003).

[2] W. Rindler, Relativity (Oxford: University Press, 2006).

[3] E. Schroedinger, Annalen der Physik 382, 325 (1925); For an English translation see: J. B. Barbour and H. Pfister, eds., Mach's Principle: From Newton's Bucket to Quantum Gravity. (Birkenhauser, Boston, 1995).

[4] F. Melia and A. S. H. Shevchuk,
MNRAS 419, 2579 (2012).

[5] D. Layzer, Astronomical Journal 59, 268 (1954).

[6] D. W. Sciama, MNRAS 113, 34 (1953).

[7] G. Berkeley, De Motu.(1721) (English translation by Arthur Aston Luce. Nelson, London, 1951).

[8] R. Drever, Philosophical Magazine 6, 683 (1961)

[9] R. H. Dicke, Phys. Rev. Lett. 7, 359 (1961) 